\title{
Prorenin and secreted frizzled-related protein 4 levels in women with gestational diabetes mellitus
}

\author{
Baldane $\mathrm{S}^{1}$, Ipekci $\mathrm{SH}^{1}$, Kebapcilar $\mathrm{AG}^{2}$, Abusoglu $\mathrm{A}^{3}$, Beyhekim $\mathrm{H}^{2}$, Ilhan $\mathrm{TT}^{2}$, Unlu A ${ }^{3}$, \\ Kebapcilar $\mathrm{A}^{1}$ \\ Division of Endocrinology and Metabolism, Department of Internal Medicine, Faculty of Medicine, \\ Selcuk University, Konya, Turkey. baldane42@hotmail.com
}

\begin{abstract}
OBJECTIVE: This study was designed to investigate prorenin and secreted frizzled-related protein 4 (SFRP4) levels in pregnancies with or without gestational diabetes mellitus (GDM).

METHODS: A total of 76 pregnant women were included in the study. Thirty-five of the pregnant women were included in GDM group according to the results of oral glucose tolerance tests (OGTT) and 41 of them were included in the control group.

RESULTS: In the group with GDM, SFRP4 value was found to be significantly higher than that of the control group $(5.59 \pm 3.32 \mathrm{ng} / \mathrm{mL}$ vs $4.05 \pm 2.15 \mathrm{ng} / \mathrm{mL} ; \mathrm{p}=0.017)$. Women with GDM had significantly higher serum prorenin levels compared with control group [737 (427-1339) pg/mL vs. 535 (376-725) pg/mL; $p=0.009$ ]. There was a significant positive association between prorenin and SFRP4 levels in GDM $(r=0.91 ; p<0.001)$ and control groups $(r=0.42 ; p=0.002)$ and whole pregnancies $(r=0.75 ; p=0.002)$.

CONCLUSION: We have shown that prorenin and SFRP4 were significantly elevated in GDM patients when compared to healthy control group. Furthermore, we found that there was a positive correlation between prorenin and SFRP4 (Tab. 1, Fig. 2, Ref. 38). Text in PDF www.elis.sk.

KEY WORDS: gestational diabetes mellitus, prorenin, SFRP 4, subclinical inflammation.
\end{abstract}

\section{Introduction}

Women with gestational diabetes mellitus (GDM) are characterized by increased serum angiotensin II (Ang II) levels. Increased prorenin levels promote pathogenesis of diabetes through Ang II poduction. Recent animal and human studies suggest that Ang II contents contributes to the development of insulin resistance in skeletal muscle tissues and induces subclinical inflammation and new-onset diabetes mellitus (1-3). In addition, the level of (pro) renin receptor was reported to be high in pregnant women with GDM compared to healthy pregnant women in a s number of studies (4-6). Having higher levels of prorenin in diabetic patients is reported by previous studies (7-10).

On the other hand, secreted frizzled-related protein 4 (SFRP-4) reduces insulin secretion and increased proinflammatory factors (11-14). Increased SFRP4 in diabetic subjects can induce IL-1 $\beta$ concentrations (15). SFRP-4 is also expressed in the placenta

${ }^{1}$ Division of Endocrinology and Metabolism, Department of Internal Medicine, Faculty of Medicine, Selcuk University, Konya, Turkey, ${ }^{2}$ Department of Obstetrics and Gynaecology, Faculty of Medicine, Selcuk University, Konya, Turkey, and ${ }^{3}$ Department of Biochemistry, Faculty of Medicine, Selcuk University, Konya, Turkey

Address for correspondence: S. Baldane, Dr, Division of Endocrinology and Metabolism, Department of Internal Medicine, Faculty of Medicine, Selcuk University, Konya, 42075, Turkey.

Phone: +903322244685, Fax: +903322412184

Acknowledgments: This study has been partially supported by Selçuk University under grant number BAP 15401082.
(16-18). Increasing evidence suggests that the increase in proinflammatory factors plays an important factor in the development of GDM (19-21). Therefore, an elevation of SFRP-4 may be a good marker of beta cell dysfunction and it can play an important risk factor in the pathogenesis of GDM.

To our knowledge SFRP-4 levels have never been investigated in patients with GDM. The aim of this study was to compare prorenin and SFRP-4 levels in pregnant women with GDM and healthy pregnant women and to evaluate the association between the levels of prorenin and SFRP-4.

\section{Materials and methods}

\section{Subjects}

The present study was a cross-sectional, case-control study. All patients participating in the study signed an informed consent form. The study was approved by our institutional ethics committee.

All recruited pregnant women were selected randomly from women admitted to our gynecology and endocrinology outpatient clinic between January 2015 and June 2016. Women with a twin pregnancy, prior cardiovascular disease, pregestational diabetes, those taking any medication or suffering from gestational or preexisting hypertensive disorders, renal or thyroid diseases, smokers, and those with acute or chronic infections were excluded.

Oral glucose tolerance test (OGTT) was performed in all pregnant women included in the study between weeks 24 and 28 of pregnancy using $75 \mathrm{~g}$ of glucose. Pregnant women were diagnosed to have GDM when one or more of the test results were abnormal 
according to the recommendations of American Diabetes Association (ADA; fasting $\geq 92 \mathrm{mg} / \mathrm{dL}, \geq 180 \mathrm{mg} / \mathrm{dL}$ after $1 \mathrm{~h}$, and $\geq 153$ $\mathrm{mg} / \mathrm{dL}$ after $2 \mathrm{~h} ; 22$ ). Age, gestational age and body mass index of all pregnant women were recorded.

\section{Blood sampling and assay}

The blood samples were allowed to clot at room temperature and then centrifuged at room temperature for $10 \mathrm{~min}$ at 2,000 g. All tubes were stored at $-80^{\circ} \mathrm{C}$ until analysis.

Serum glucose, total cholesterol, triglycerides and HDL-cholesterol levels were measured using Abbott Architect C-8000. LDL-cholesterol was derived by the Friedewald equation method. Glucose levels were measured using a hexokinase method and Abbott Architect C-8000 device.

\section{SFRP-4 and prorenin assay}

Serum SFRP-4 levels were measured using specific enzyme-linked immunosorbent assay (ELISA) immunoassay kit (Mybiosource, San Diego, CA, USA). Serum prorenin concentrations were measured using an ELISA kit (LINCO Research Inc., St. Charles, MO, USA).

\section{Statistical analysis}

The Kolmogorov-Smirnov normality test was used to determine the distribution pattern of the variables. Results were presented as mean \pm standard deviation. A comparison between groups of continuous variables was performed using a Student's $\mathrm{t}$ test or a Mann-Whitney U test. Differences between categorical variables were analyzed using a Fisher's exact test. Relationships between variables were analyzed using Pearson's or Spearman's rank correlation coefficients. Binary logistical regression analysis was used to identify the factors associated with the presence of GDM. The statistical analysis was carried out using the Statistical Package for the Social Sciences (SPSS), version 20.0 (SPSS Inc., Chicago, IL, USA). A p-value of $<0.05$ was considered statistically significant.

\section{Results}

A total of 76 pregnant women were included in the study. Thirty-five of the pregnant women were included in the GDM group according to the results of OGTT, and 41 of them were included in the control group.

There were no significant differences in age, gestational age, BMI and lipid profile levels between the two groups (Tab. 1). However fasting blood glucose (FBG), prorenin and SFRP-4 were significantly higher in individuals with GDM (Tab. 1). The mean FBG level of GDM group was $91.54 \pm 15.11 \mathrm{mg} / \mathrm{dL}$ and mean FBG level of control group was $77.94 \pm 5.88 \mathrm{mg} / \mathrm{dL}$ ( $\mathrm{p}<0.001)$.

In the group with GDM, SFRP4 value was found to be significantly higher than that of the control group $(5.59 \pm 3.32 \mathrm{ng} / \mathrm{mL}$ vs $4.05 \pm 2.15 \mathrm{ng} / \mathrm{mL} ; \mathrm{p}=0.017$ ). Women with GDM had significantly higher serum prorenin levels compared with the control group [737 (427-1339) pg/mL vs. 535 (376-725) pg/mL; $\mathrm{p}=0.009]$.
Tab. 1. Clinical and laboratory characteristics of GDM and control groups.

\begin{tabular}{lccc}
\hline Parameter & GDM $(\mathrm{n}=35)$ & Control $(\mathrm{n}=41)$ & $\mathrm{p}$ \\
\hline Age (years) & $30.11 \pm 4.26$ & $29.14 \pm 4.83$ & 0.3 \\
Gestational age (weeks) & $26.04 \pm 4.66$ & $25.23 \pm 3.75$ & 0.652 \\
BMI $\left(\mathrm{kg} / \mathrm{m}^{2}\right)$ & $29.07 \pm 3.26$ & $28.33 \pm 4.01$ & 0.1 \\
FBG $(\mathrm{mg} / \mathrm{dL})$ & $91.54 \pm 15.11$ & $77.94 \pm 5.88$ & $<0.001$ \\
Total cholesterol (mg/dL) & $240.10 \pm 48.35$ & $230.72 \pm 41.30$ & 0.3 \\
LDL cholesterol $(\mathrm{mg} / \mathrm{dL})$ & $135.81 \pm 46.13$ & $128.27 \pm 31.71$ & 0.4 \\
HDL cholesterol (mg/dL) & $61.04 \pm 13.25$ & $61.31 \pm 10.51$ & 0.9 \\
Triglycerides $(\mathrm{mg} / \mathrm{dL})$ & $229.50 \pm 76.33$ & $194.85 \pm 69.21$ & 0.05 \\
Prorenin $(\mathrm{pg} / \mathrm{mL})$ & $737(427-1339)$ & $535(376-725)$ & 0.009 \\
SFRP- $4(\mathrm{ng} / \mathrm{mL})$ & $5.59 \pm 3.32$ & $4.05 \pm 2.15$ & 0.017 \\
\hline GDP
\end{tabular}

GDM - gestational diabetes mellitus, BMI - body mass index, FBG - fasting blood glucose, LDL - low-density lipoprotein, HDL - high-density lipoprotein

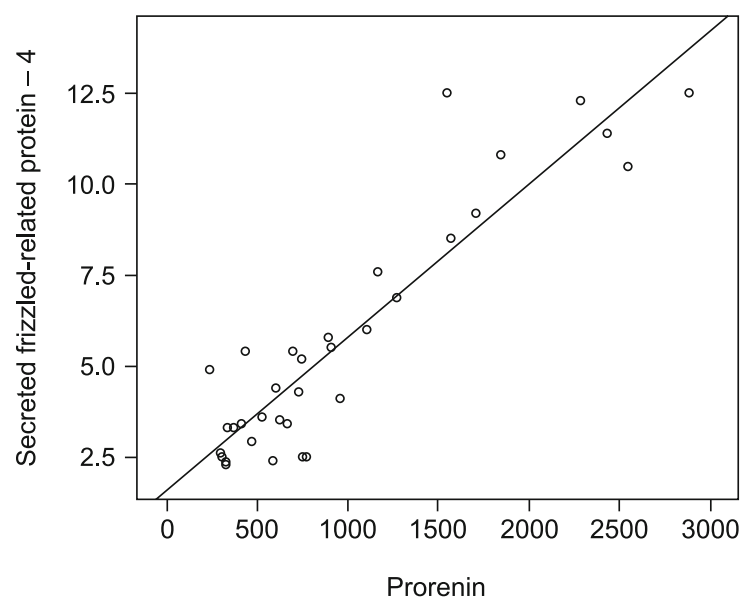

Fig. 1. Correlation between prorenin and SFRP4 levels in GDM.

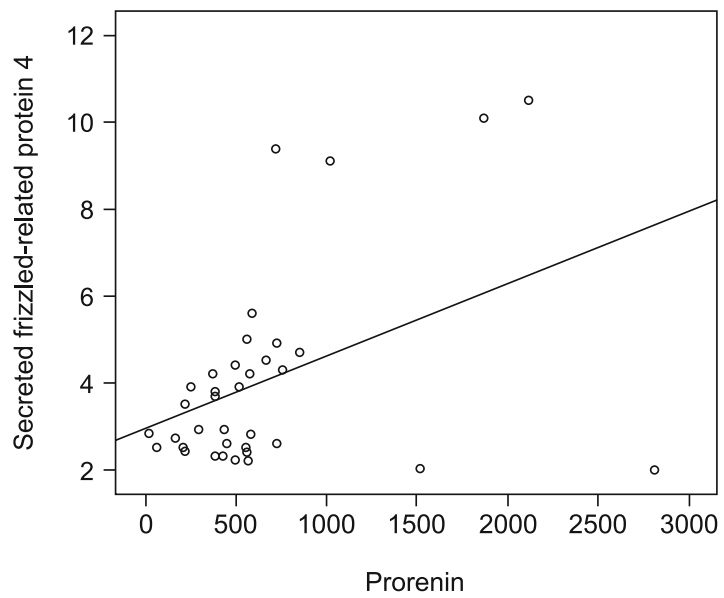

Fig. 2. Correlation between between prorenin and SFRP4 levels in the control group.

There was a significant positive association between prorenin and SFRP4 levels in GDM $(r=0.91 ; \mathrm{p}<0.001)$ (Fig. 1) and control groups $(\mathrm{r}=0.42 ; \mathrm{p}=0.002)$ (Fig. 2$)$ and whole whole pregnancies $(\mathrm{r}=0.75 ; \mathrm{p}=0.002)$. No other feature was significantly associated with prorenin and SFRP-4. 
$450-453$

A significant independent association was found between plasma SFRP-4 and GDM in the binary logistic regression analysis after adjusting for age, BMI, and lipid profile [odds ratio (OR): $5.872 ; 95 \%$ confidence interval $(\mathrm{CI}) ; \mathrm{p}=0.015]$. A significant independent association was found between plasma prorenin and GDM in the binary logistic regression analysis after adjusting for age, BMI, and lipid profile (OR: 4.182; 95\% CI; $\mathrm{p}=0.041$ ).

\section{Discussion}

In the present study, prorenin and SFRP-4 concentrations were higher in women with GDM and a positive association between the prorenin and SFRP-4 in GDM and non-GDM women was exhibited.

The relationship between prorenin receptor (4-6), prorenin levels (7-10), and diabetes mellitus has been demonstrated in previous studies. Similarly, we found a higher concentration of prorenin levels in GDM group when compared to non-GDM group. Increased plasma prorenin activity has also been described in subjects who had diabetes with complications $(8,23,24)$. The association of diabetic organ injury and increase in plasma prorenin has not been clearly established yet. Prorenin was demonstrated to initiate an inflammation via extracellular signal-related protein kinases 1 and 2 (ERK 1/2) and increased mitogen-activated protein kinase activity (25-26). Thus MAP kinases are modulating IRS-1 and GLUT4 protein levels. In humans, decreased GLUT4 and IRS-1 protein abundance can induce the development of type 2 diabetes mellitus (27-28).

Nagai et al. investigated that the increase in (pro)renin receptor in skeletal muscle promotes the development of insulin resistance in animal model (29). Rafiq et al. reported that (pro)renin receptor could induce a decrease in insulin secretion by causing beta-cell dysfunction in the pancreas (30). Recently, one of the studies found that an increase in prorenin levels during the first trimester may induce the development of GDM (6). Bokuda et al. reported that prorenin may contribute to the development of $\beta$-cell dysfunction through RAS, ERK 1/2 and MAP kinases activations (4). Wnt- $\beta$ catenin pathway is involved in diabetes mellitus (31). Recently, (pro)renin receptor was determined to be an accessory subunit for vacuolar (V-ATPase), which contributes to the activation of the canonical Wnt- $\beta$-catenin signaling pathway (32). WNT/ $\beta$-catenin is activated in epithelial cells, along with significant increases in IL-1 $\beta$ levels (33). Our study results suggest that a high prorenin levels may be a potential risk factor for GDM.

On the other hand, SFRP-4 levels were higher in women with GDM. Our study revealed a relationship between SFRP-4 and prorenin. Mahdi et al. observed high overexpression of SFRP-4 in islets of diabetic subjects and its relationship with inflammatory markers, particularly IL- $1 \beta$ stimulating its production (15). Mahdi et al (15) and Bergmann $\mathrm{K}$ et al (14) reported that high expression of SFRP-4 was associated with inflammation and decreased insulin secretion, therefore it seems to be serving as a biomarker of the pancreatic islet dysfunction in T2DM. Increased SFRP-4 concentrations suggest that SFRP-4 could be used potentially as indicators of risk factor for developing glucose intolerance in GDM women. SFRP-4 has been variably reported to activate or inhibit Wnt signaling in different tissues (33-35).

SFRP-4 levels were positively correlated with prorenin levels in GDM and non-GDM groups. It is not surprising that prorenin can induce the same proinflamatory factors such as WNT/b-catenin signaling pathway, RAS, ERK 1/2, IL-1 $\beta$, and MAP kinases activations, as well as SFRP-4. The proinflammatory cytokines IL-1b function as novel WNT/b-catenin targets. IL-1 $\beta$ indirectly activates canonical Wnt signaling by up-regulation of Wnt ligands. Overexpression of WNT/b-catenin led to acute inflammation and IL-1 $\beta$ up-regulates inflammation through activation of the NF kappa B pathway (36-38).

Some limitations should be considered when our results are interpreted. WNT/b-catenin signaling pathway, IL-1 $\beta$ activations and other inflammation markers could be performed to correlate these markers with the risk of diabetes mellitus in women with GDM.

\section{Conclusion}

We demonstrated an increase in prorenin and SFPR 4 in patients with GDM. Early intervention in GDM is important because it can improve the maternal glucose intolerance while prorenin and SFRP-4 may be important GDM predictors.

\section{References}

1. Jandeleit-Dahm KA, Tikellis C, Reid CM, Johnston CI, Cooper ME. Why blockade of the renin-angiotensin system reduces the incidence of new-onset diabetes. J Hypertens 2005; 23: 463-473.

2. Nagai Y, Ichihara A, Nakano D, et al. Possible contribution of the nonproteolytic activation of prorenin to the development of insulin resistance in fructose-fed rats. Exp Physiol 2009; 94: 1016-1023.

3. Tahmasebi M, Puddefoot JR, Inwang ER, Vinson GP. The tissue renin-angiotensin system in human pancreas. J Endocrinol 1999; 161: $317-322$.

4. Bokuda K, Ichihara A. Possible contribution of (pro)renin receptor to development of gestational diabetes mellitus. World J Diabetes 2014; 5: 912-916.

5. Gokulakrishnan K, Maheswari K, Mahalakshmi MM, et al. Association of Soluble (PRO) Renin Receptor with Gestational Diabetes Mellitus. Endocr Pract 2015; 21: 7-13.

6. Watanabe N, Morimoto S, Fujiwara T, et al. Prediction of gestational diabetes mellitus by soluble (pro)renin receptor during the first trimester. J Clin Endocrinol Metab 2013; 98: 2528-2535.

7. Tsai TJ, Tai TY. Plasma prorenin and renin levels in non-insulin-dependent diabetes mellitus. J Formos Med Assoc 1992; 91: 775-779.

8. Franken AA, Derkx FH, Man in't Veld AJ, et al. High plasma prorenin in diabetes mellitus and its correlation with some complications. J Clin Endocrinol Metab 1990; 71: 1008-1015.

9. Wilson DM, Luetscher JA. Plasma prorenin activity and complications in children with insulin-dependent diabetes mellitus. N Engl J Med 1990; 323: 1101-1106.

10. Naruse M, Wasada T, Naruse K, Yoshimoto T, Omori Y, Demura H. Pathophysiological significance of plasma total renin and prorenin in patients with diabetes mellitus. Endocr J 1995; 42: 225-233. 
11. Anand K, Vidyasagar S, Lasrado I, et al. Secreted Frizzled-Related Protein 4 (SFRP4): A Novel Biomarker of $\beta$-Cell Dysfunction and Insulin Resistance in Individuals With Prediabetes and Type 2 Diabetes. Diabetes Care 2016; 39: 147-148.

12. Brix JM, Krzizek EC, Hoebaus C, Ludvik B, Schernthaner G, Schernthaner GH. Secreted Frizzled-Related Protein 4 (SFRP4) is Elevated in Patients with Diabetes Mellitus. Horm Metab Res 2016; 48: 345-348.

13. Liu F, Qu H, Li Y, et al. Relationship between serum secreted frizzledrelated protein 4 levels and the first-phase of glucose-stimulated insulin secretion in individuals with different glucose tolerance. Endocr J 2015; 62: 733-740.

14. Bergmann K, Sypniewska G. Secreted frizzled-related protein 4 (SFRP4) and fractalkine (CX3CL1) - Potential new biomarkers for $\beta$-celldysfunction and diabetes. Clin Biochem 2014; 47: 529-532.

15. Mahdi T, Hänzelmann S, Salehi A, et al. Secreted frizzled-related protein 4 reduces insulin secretion and is overexpressed in type 2 diabetes. Cell Metab 2012; 16: 625-633.

16. White L, Suganthini G, Friis R, Dharmarajan A, Charles A. Expression of secreted frizzled-related protein 4 in the primate placenta. Reprod Biomed Online 2009; 18: 104-110.

17. Fujita M, Ogawa S, Fukuoka H, et al. Differential expression of secreted frizzled-related protein 4 in decidual cells during pregnancy. J Mol Endocrinol 2002; 28: 213-223.

18. Hewitt DP, Mark PJ, Dharmarajan AM, Waddell BJ. Placental expression of secreted frizzled related protein-4 in therat and the impact of glucocorticoid-induced fetal and placental growth restriction. Biol Reprod 2006; 75: 75-81.

19. Vitoratos N, Valsamakis G, Mastorakos G, et al. Pre- and early post-partum adiponectin and interleukin-1 beta levels in women with and without gestational diabetes. Hormones (Athens) 2008; 7: 230-236.

20. Aktulay A, Engin-Ustun Y, Ozkan MS, et al. Gestational Diabetes Mellitus Mellitus seems to be associated with inflammation. Acta Clin Croat 2015; 54: 475-478.

21. Bossick AS, Peters RM, Burmeister C, Kakumanu N, Shill JE, Cassidy-Bushrow AE. Antenatal inflammation and gestational diabetes mellitus risk among pregnant African-American women. J Reprod Immunol 2016; 115: 1-5.

22. American Diabetes Association. Standards of medical care in diabetes - 2011. Diabetes Care. 2011; 34: 11-61.

23. Luetscher JA, Kraemer FB, Wilson DM. Prorenin and vascular complications of diabetes. Am J Hypertens 1989; 2: 382-386.

24. Wilkinson-Berka JL. Prorenin and the (pro)renin receptor in ocular pathology. Am J Pathol 2008; 173: 1591-1594.

25. Feldt S, Batenburg WW, Mazak I, et al. Prorenin and renin-induced extracellular signal-regulated kinase $1 / 2$ activation in monocytes is not blocked by aliskiren or the handle-region peptide. Hypertension 2008; 51: 682-688.
26. Sakoda M, Ichihara A, Kaneshiro Y, et al. (Pro)renin receptor-mediated activation of mitogen-activated protein kinases in human vascular smooth muscle cells. Hypertens Res 2007; 30: 1139-1146.

27. Carvalho E, Jansson P-A, Axelsen M, et al. Low cellular IRS-1 gene and protein expression predict insulin resistance and NIDDM. Faseb J 1999; 13: 2173-2178.

28. Carvalho E, Jansson P-A, Nagaev I, Wenthzel A-M, Smith U. Insulin resistance with low cellular IRS-1 expression is also associated with low GLUT4 expression and impaired insulin-stimulated glucose transport. Faseb J 2001; 15: 1101-1103.

29. Nagai Y, Ichihara A, Nakano D, et al. Possible contribution of the non-proteolytic activation of prorenin to the development of insulin resistance in fructose-fed rats. Exp Physiol 2009; 94: 1016-1023.

30. Rafiq K, Hitomi H, Nakano D, Ichihara A, Nishiyama A. Possible involvement of the (pro)renin receptor-dependent system in the development of insulin resistance. Front Biosci (Schol Ed) 2011; 3: 1478-1485.

31. Kato H, Gruenwald A, Suh JH, et al. Wnt/beta-catenin pathway in podocytes integrates cell adhesion, differentiation, and survival. J Biol Chem 2011; 286: 26003-26015.

32. Cruciat CM, Ohkawara B, Acebron SP, et al. Requirement of prorenin receptor and vacuolar $\mathrm{H}+-$ ATPase-mediated acidification for Wnt signaling. Science 2010; 327: 459-463.

33. Lee SH, Demeterco C, Geron I, Abrahamsson A, Levine F, Itkin Ansari P. Islet specific Wnt activation in human type II diabetes. Exp. Diabetes Res 2008; 2008: 728763.

34. Suzuki H, Watkins DN, Jair KW, et al. Epigenetic inactivation of SFRP genes allows constitutive WNT signaling in colorectal cancer. Nat Genet 2004; 36: 417-422.

35. Carmon KS, Loose DS. Secreted frizzled-related protein 4 regulates two Wnt7a signaling pathways and inhibits proliferation in endometrial cancer cells. Mol Cancer Res 2008; 6: 1017-1028.

36. Aumiller V, Balsara N, Wilhelm J, Günther A, Königshoff M. WNT/ $\beta$-catenin signaling induces IL-1 $\beta$ expression by alveolar epithelial cells in pulmonary fibrosis. Am J Respir Cell Mol Biol 2013; 49: 96-104.

37. Ma B, van Blitterswijk CA, Karperien M. A Wnt/ß-catenin negative feedback loop inhibits interleukin-1-induced matrix metalloproteinase expression in human articular chondrocytes. Arthritis Rheum 2012; 64: 2589-2600.

38. Sonomoto K, Yamaoka K, Oshita K, et al. Interleukin- $1 \beta$ induces differentiation of human mesenchymal stem cells into osteoblasts via the Wnt-5a/receptor tyrosine kinase-like orphan receptor 2 pathway. Arthritis Rheum 2012; 64: 3355-3363. 